\title{
Progresso científico sobre nutrição de animais de companhia na primeira década do século XXI
}

\author{
Aulus Cavalieri Carciofi ${ }^{1}$, Juliana Toloi Jeremias ${ }^{2}$ \\ ${ }^{1}$ Docente do Departamento de Clínica e Cirurgia Veterinária, Faculdade de Ciências Agrárias e Veterinárias, Universidade Estadual Paulista, \\ campus de Jaboticabal. Via de Acesso Prof. Paulo Donato Castellane, s/n. Jaboticabal - SP. CEP: 14.884-900. Tel.: (16) 32092631. Fax: \\ (16) 3203-1226. \\ 2 Doutoranda da Faculdade de Ciências Agrárias e Veterinárias, Universidade Estadual Paulista, campus de Jaboticabal. Via de Acesso Prof. \\ Paulo Donato Castellane, s/n. Jaboticabal - SP. CEP: 14.884-900. Tel.: (16) 32092631. Fax: (16) 3203-1226.
}

RESUMO - O conhecimento científico sobre nutrição de animais de companhia tem aumentado de forma contínua, acompanhando o fenômeno visto em diversas áreas de conhecimento. Na última década, têm-se prioritariamente pesquisas direcionadas ao uso de nutrientes na promoção de saúde, prevenção de doenças degenerativas, melhoria da qualidade de vida e aumento da expectativa de vida de cães e gatos. Este direcionamento de pesquisas é, em grande parte, explicado pela importância que cães e gatos assumiram na vida das pessoas, fazendo com que as decisões alimentares dos proprietários com seus animais se assemelhassem às que adotam para si próprios. A publicação da nova revisão do Nutrient Requirements of Dogs and Cats em 2006 foi, talvez, o avanço recente mais significativo, trazendo novo entendimento sobre necessidades energéticas e de nutrientes nas diferentes fases de desenvolvimento e estados fisiológicos. Apesar destes avanços, ainda são necessárias pesquisas na área de caracterização físico-química e de utilização dos ingredientes, efeitos do processo de extrusão e mesmo de necessidades nutricionais, que conta com uma base pequena de artigos disponíveis, a maioria antiga. Talvez os desafios científicos mais importantes em nutrição de cães e gatos sejam metabolismo de carboidratos, importância da massa corporal magra na saúde, urolitíases, gerontologia, relação entre microbiota intestinal e saúde, imunonutrição e manejo nutricional em condições clínicas específicas importantes para estas espécies.

Palavras-chave: cães, doenças nutricionais, gatos, necessidades nutricionais, nutrientes

\section{Scientific progress on companion animal nutrition on the first decade of the Century XXI}

\begin{abstract}
Scientific knowledge about companion animal nutrition has increased incrementally, following the phenomenon seen in several areas of knowledge. In the last decade may be the priority of research was directed to the use of nutrients in health promotion, prevention of degenerative diseases, improve quality of life and increased life expectancy of dogs and cats. This direction of research is largely explained by the importance that dogs and cats taken in people's lives, making that the decisions of the owners about the nutrition of their animals resembled those which adopt for themselves. The publication of the new revision of Nutrient Requirements of Dogs and Cats in 2006 was perhaps the most significant recent development, bringing new understanding of energy and nutrient needs at different developmental stages and physiological states. Despite these advances, further research is needed in the area of physical-chemical characterization and use of ingredients, effects of the extrusion process and even nutritional needs, which has a small base of available articles, many of them quite old. Perhaps the most important scientific challenge in nutrition of dogs and cats are carbohydrate metabolism, the importance of lean body mass in health, gerontology, the relationship between intestinal microbiota and health, immunonutrition and the nutritional management of important clinical conditions specific to these animals.
\end{abstract}

Key Words: cats, dogs, nutrients, nutritional diseases, nutritional requirements

\section{Introdução}

Pesquisas científicas relacionadas à nutrição de animais de companhia, principalmente nos últimos 10 anos, deixaram de focar a dicotomia necessidades mínimas e teores máximos, sobretudo quanto ao estabelecimento das recomendações nutricionais. O conhecimento das necessidades mínimas deixou de ser tão importante e, cada vez mais, busca-se entender o papel da nutrição na promoção de saúde, bem-estar e longevidade. A adequação da composição nutricional, matriz de ingredientes, processamento do alimento, entre outros, às necessidades específicas de cada estágio de vida, à condição fisiológica, ao estilo de vida e às diversas condições fisiopatológicas

Corresponding author: aulus.carciofi@gmail.com 
que acometem estes animais têm ocupado os temas principais de pesquisa.

Este novo direcionamento de objetivos científicos é em grande parte explicado pela mudança da condição de experimentação e importância que cães e gatos assumiram na sociedade. No início da pesquisa estes eram encarados como animais de laboratório, usados em protocolos que, embora adaptados à sua fisiologia e necessidades comportamentais específicas, em pouco se diferenciavam dos empregados em roedores e outros animais de pesquisa. Hoje inseridos na estrutura familiar, as informações científicas que resultam em impacto social e econômico requerem protocolos, métodos e propósitos de investigação totalmente diferentes, que se aproximam bastante da própria dinâmica investigativa da nutrição humana. Recebendo informações nutricionais da mídia, proprietários passaram a se interessar também em como a alimentação poderia afetar a saúde de seus animais. A partir desta demanda pesquisadores e indústria de alimentos para cães e gatos têm respondido, redirecionando objetivos e métodos de pesquisa. A pesquisa nutricional tem, assim, se diversificado em objetivos abrangendo áreas como longevidade, gerontologia, bem-estar, imunidade, beleza de pele e pelos, função digestiva, função cognitiva, saúde oral e prevenção de doenças degenerativas, dentre as quais pode-se incluir o manejo nutricional de extenso número de condições como urolitíases, neoplasias, cardiopatias, nefropatias, artropatias, endocrinopatias, obesidade, distúrbios gastrointestinais, distúrbio alérgicos, entre outros.

Paralelo a toda esta pesquisa em saúde, verificam-se alguns estudos sobre ingredientes e muito poucos sobre processo, aspectos não tão bem conhecidos para cães e gatos e que necessitariam de mais investigação. Este texto traz breve revisão sobre algumas destas novas abordagens em pesquisa, não necessariamente destacando os mais importantes ou mais extensamente investigados, mas também àqueles que são mais familiares aos autores desta revisão. Novos pontos de vista sobre as recomendações nutricionais

No início desta década foi formado um comitê pelo Conselho Nacional de Pesquisas Norte Americano para revisar as publicações sobre necessidades nutricionais de cães e gatos, anteriormente reunidas no Nutrient Requirements of Dogs (1985) e no Nutrient Requirements of Cats (1986). Foi atribuída a esse comitê a tarefa de revisar estas publicações e transformá-las em um único documento, que veio a ser publicado em 2006 (NRC, 2006). Antes do lançamento do NRC (2006) haviam apenas informação dispersas sobre a biodisponibilidade de nutrientes e necessidades nutricionais de animais de companhia. O progresso na nutrição de cães e gatos exigia informações mais precisas sobre as necessidades das diversas fases da vida (especialmente a reprodução e manutenção), juntamente com os valores de biodisponibilidade dos nutrientes dos ingredientes das dietas (Morris \& Rogers, 2004).

O Comitê analisou e sintetizou milhares de artigos científicos publicados nos últimos 25 anos. Fez recomendações nutricionais baseadas no nível de atividade física e estágio de vida do animal. A publicação também analisa a forma como os nutrientes são metabolizados, os indícios de deficiência nutricional e doenças relacionadas à má nutrição. Há discussão abrangente dos aditivos alimentares para animais de estimação e tabelas detalhando a composição dos ingredientes normalmente utilizados, em texto de referência para pesquisadores, fabricantes de alimentos e os veterinários. Deixando clara a preocupação com a qualidade de vida, longevidade e até mesmo com a obesidade, a última edição do NRC (2006) estabeleceu novas necessidades energéticas diárias para cães e gatos e dividiu as necessidades dos nutrientes em quatro categorias distintas, diferente das versões anteriores (NRC, 1985, 1986) que apresentavam apenas as necessidades mínimas e máximas. As novas categorias criadas estão descritas a seguir:

$\S$ Necessidades mínimas (NM) - representa a concentração ou quantidade mínima biodisponível do nutriente que supre as necessidades em um determinado estado fisiológico.

$\S$ Ingestão adequada(IA) - representa a concentração ou a quantidade de um nutriente que supre as necessidades em uma determinada fase da vida, estimada nos casos em que não foram determinadas experimentalmente as NM. É baseada em dados de estudos sobre o assunto já publicados com cães e gatos, e, em alguns casos, em dados comparativos retirados de estudos em outras espécies.

$\S$ Ingestão recomendada (IR) - expressão do inglês “recommended allowance”, representa a concentração ou quantidade recomendada de um nutriente em dieta prática formulada para um determinado estado fisiológico. A IR é baseada na NM e, se for caso, inclui fator de segurança para nutrientes com biodisponibilidade não determinada. Quando não existe NM, a IR é baseada na IA.

$\S$ Limite superior de segurança (LSS) - valores baseados na concentração ou quantidade máxima de nutrientes que não está associada a efeitos adversos em cães e gatos. Infelizmente, os valores de LSS não estão disponíveis para muitos nutrientes, especialmente para minerais e vitaminas.

Evidencia-se, assim, um enfoque próximo ao da nutrição humana no novo texto do NRC (2006). Mesmo existindo 
muitas lacunas sobre as necessidades nutricionais mínimas, estas foram contornadas pela proposição das IR. Os nutrientes que ainda carecem de estudos para estabelecimento de suas NM estão listados na Tabela 1.

Destacam-se, em termos de IR, a inclusão dos ácidos graxos aracdônico, eicosapentaenóico e docosahexaenoico, tanto para cães como para gatos (Bauer, 2008). Desta forma, a semelhança da formulação de aminoácidos, a formulação de ácidos graxos exige a inclusão de vários ingredientes com vistas à obtenção de concentrações específicas destes nutrientes para cada espécie ou faixa etária.

\section{Informação sobre digestibilidade de ingredientes}

O conhecimento sobre biodisponibilidade dos nutrientes nos alimentos empregados nas formulações evoluiu pouco nos últimos anos (Bontempo, 2005, Carciofi et al., 2006). A produção destas informações, inclusive, vem se reduzindo recentemente devido ao maior enfoque em saúde das pesquisas mais recentes (Carciofi et al., 2009). Mesmo métodos de estudo da digestibilidade dos alimentos é área pouco pesquisada para cães (Carciofi et al., 2007) e gatos (Vasconcellos et al., 2006; Vasconcellos et al., 2007). A escassez de informações faz com que, via de regra, as formulações para animais de companhia sejam baseadas nos nutrientes brutos, não nos disponíveis (Fortes et al., 2010), indicando a necessidade de pesquisas na área de caracterização físico-química dos ingredientes utilizados pela indústria, de forma a aperfeiçoar a utilização destes nas formulações (Fahey, 2003; Carciofi, 2008). Talvez por isto, ao menos no Brasil, produtos com problemas de composição de rótulo (Carciofi et al., 2006) e com baixa digestibilidade e energia metabolizável ainda estejam disponíveis no mercado (Carciofi et al., 2009).

Tabela 1 - Nutrientes que ainda necessitam de estudo para determinação de suas necessidades mínimas (NM) para cães e gatos em diferentes estágios de vida e estados fisiológicos

\begin{tabular}{|c|c|c|c|c|c|}
\hline \multirow[b]{2}{*}{ Nutriente } & \multicolumn{5}{|c|}{ Necessidades mínimas ainda não determinadas* } \\
\hline & $\begin{array}{c}\text { Cães crescimento } \\
\text { desmame }\end{array}$ & Cães manutenção & Gatos crescimento & $\begin{array}{c}\text { Gatos } \\
\text { manutenção }\end{array}$ & $\begin{array}{c}\text { Gatas gestação e } \\
\text { pico lactação }\end{array}$ \\
\hline Aminoácidos & - & - & - & $\begin{array}{l}\text { Arginina } \\
\text { Histidina } \\
\text { Isoleucina } \\
\text { Leucina } \\
\text { Fenilalanina } \\
\text { Tirosina } \\
\text { Treonina } \\
\text { Triptofano } \\
\text { Valina }\end{array}$ & $\begin{array}{c}\text { Arginina } \\
\text { Histidina } \\
\text { Isoleucina } \\
\text { Metionina } \\
\text { Metionina+Cistina } \\
\text { Leucina } \\
\text { Lisina } \\
\text { Fenilalanina } \\
\text { Treonina } \\
\text { Triptofano } \\
\text { Valina }\end{array}$ \\
\hline Ácidos graxos & $\begin{array}{c}\text { Linoleico } \\
\alpha \text {-linolênico } \\
\text { Araquidônico } \\
\text { Eicosapentaenoico } \\
\text { Docosahexaenoico }\end{array}$ & $\begin{array}{c}\text { Linoleico } \\
\alpha \text {-linolênico } \\
\text { Araquidônico } \\
\text { Eicosapentaenoico } \\
\text { Docosahexaenoico }\end{array}$ & $\begin{array}{c}\text { Linoleico } \\
\alpha \text {-linolênico } \\
\text { Araquidônico } \\
\text { Eicosapentaenoico } \\
\text { Docosahexaenoico }\end{array}$ & $\begin{array}{c}\text { Linoleico } \\
\alpha \text {-linolênico } \\
\text { Araquidônico } \\
\text { Eicosapentaenoico } \\
\text { Docosahexaenoico }\end{array}$ & $\begin{array}{c}\text { Linoleico } \\
\alpha \text {-linolênico } \\
\text { Araquidônico } \\
\text { Eicosapentaenoico } \\
\text { Docosahexaenoico }\end{array}$ \\
\hline Minerais & $\begin{array}{c}\mathrm{P}, \mathrm{Na}, \mathrm{K}, \mathrm{Cl}, \\
\mathrm{Cu}, \mathrm{Mn}, \mathrm{I}\end{array}$ & $\begin{array}{c}\text { P, K, Cl, Fe, } \\
\mathrm{Cu}, \mathrm{Zn}, \mathrm{Mn}, \mathrm{Se}\end{array}$ & Mn, I & $\begin{array}{c}\mathrm{K}, \mathrm{Cl}, \mathrm{Fe}, \mathrm{Cu}, \\
\mathrm{Zn}, \mathrm{Mn}, \mathrm{Se}\end{array}$ & $\begin{array}{c}\mathrm{Ca}, \mathrm{Na}, \mathrm{K}, \mathrm{Cl}, \mathrm{Fe}, \\
\mathrm{Cu}, \mathrm{Mn}, \mathrm{Se}, \mathrm{I}\end{array}$ \\
\hline Vitaminas & $\begin{array}{c}\text { Vitamina A } \\
\text { Colecalciferol } \\
\text { Vitamina E } \\
\text { Vitamina K } \\
\text { Tiamina } \\
\text { Riboflavina } \\
\text { Niacina } \\
\text { Ácido pantotênico } \\
\text { Biotina } \\
\text { Ácido fólico } \\
\text { Cobalamina } \\
\text { Colina }\end{array}$ & $\begin{array}{c}\text { Vitamina A } \\
\text { Colecalciferol } \\
\text { Vitamina E } \\
\text { Vitamina K } \\
\text { Tiamina } \\
\text { Niacina } \\
\text { Ácido pantotênico } \\
\text { Piridoxina } \\
\text { Biotina } \\
\text { Ácido fólico } \\
\text { Cobalamina } \\
\text { Colina }\end{array}$ & $\begin{array}{l}\text { Vitamina A } \\
\text { Vitamina E } \\
\text { Vitamina K } \\
\text { Riboflavina } \\
\text { Niacina } \\
\text { Biotina } \\
\text { Cobalamina }\end{array}$ & $\begin{array}{c}\text { Vitamina A } \\
\text { Colecalciferol } \\
\text { Vitamina E } \\
\text { Vitamina K } \\
\text { Tiamina } \\
\text { Niacina } \\
\text { Biotina } \\
\text { Cobalamina }\end{array}$ & $\begin{array}{c}\text { Vitamina A } \\
\text { Colecalciferol } \\
\text { Vitamina E } \\
\text { Vitamina K } \\
\text { Riboflavina } \\
\text { Tiamina } \\
\text { Niacina } \\
\text { Biotina } \\
\text { Cobalamina }\end{array}$ \\
\hline
\end{tabular}

* Cadelas em gestação e pico piridoxina de lactação não apresentam NM definidas para nenhum nutriente. Adaptado de NRC (2006). 
Saúde, qualidade e expectativa de vida

Os avanços na nutrição de animais de companhia têm seguido aqueles verificados na nutrição humana. Vê-se no mundo uma explosão do número de marcas de dietas comerciais prontas para o consumo, com formulações cada vez mais sofisticadas e específicas (Steiff \& Bauer, 2001). Os conceitos de nutrição estão se expandindo para além da fronteira da sobrevivência e satisfação da fome para enfatizar a utilização de alimentos que promovam bem estar, melhora de saúde e redução do risco de doenças (Fahey, 2003). Têm se buscado compreender como a dieta pode maximizar a expectativa e a qualidade de vida pela utilização de ingredientes e nutrientes que desenvolvam a capacidade de resistir a doenças e melhorem a saúde (Tzortzis et al., 2003). Exemplo disso são estudos recentes sobre a influência da restrição energética sobre a expectativa e qualidade de vida em cães (Kealy et al., 2002), ou sobre o uso de nutrientes por felinos idosos (Teshima et al., 2010).

Nos últimos anos, as pesquisas em nutrição de animais de companhia têm também dado maior ênfase aos aspectos metabólicos desencadeados pela ingestão dos alimentos. Os efeitos metabólicos do alimento estão relacionados com alterações de saúde a longo prazo, que podem se estabelecer ao longo de vários meses ou anos de ingestão alimentar. Alguns exemplos incluem as urolitíases, nefropatias, alterações articulares, distúrbios cardio-circulatórios, obesidade, intolerância aos carboidrados (Diabetes Mellitus), dentre outras, todas relacionadas com a qualidade de vida e longevidade de cães e gatos (Carciofi, 2007).

Papel da nutrição na prevenção e suporte de afecções

O emprego de alimentos na promoção de saúde se estende por diversas áreas, como saúde bucal (Carciofi et al., 2007), suporte nutricional em condições de hospitalização (Brunetto et al., 2010) e suporte nutricional em afecções clínicas diversas (Tortola et al., 2009; Jeremias et al., 2009). Este é um tópico bastante amplo, não sendo objetivo desta revisão fazer apanhado completo sobre o mesmo. Alguns pontos com quantidade importante de pesquisas na última década seriam:

\section{Obesidade}

A obesidade está transformando-se em um dos grandes desafios dos nutricionistas e pesquisadores no século XXI (Budsberg, 2010). Esta se tornou o problema de saúde mais frequente em cães e gatos de estimação. Segundo o último levantamento da Associação Médica Veterinária Americana, 40\% dos cães dos Estados Unidos apresentam sobrepeso ou são obesos. A principal preocupação médica na obesidade é que esta está associada a muitas doenças, com efeitos prejudiciais sobre a saúde e longevidade dos cães e gatos (Michel et al., 2008). Os problemas a que os animais de companhia obesos podem estar predispostos incluem doenças ortopédicas, diabetes mellitus, anormalidades no perfil lipídico, doenças cardio-respiratórias, urinárias, distúrbios reprodutivos, neoplasias (tumores de mama, carcinoma de células de transição), doenças dermatológicas e complicações anestésicas (German, 2006). Tem sido demonstrado que a obesidade aumenta o estresse oxidativo em crianças obesas, gatos e outras espécies. O estresse oxidativo pode resultar em danos no DNA, com alterações posteriores na expressão gênica, sinalização celular, mutações, morte e transformação celular. Estes efeitos do dano oxidativo podem predispor os animais e os seres humanos a numerosas doenças, incluindo o câncer (Cline et al., 2009).

Opções terapêuticas nutricionais têm sido investigadas para cães (Carciofi et al., 2005; Brunetto et al., 2008) e gatos (Vasconcellos et al., 2009) obesos. Importante preocupação com dieta restrita de energia, no entanto, é que esta cubra todas as necessidades de nutrientes essenciais, especialmente aminoácidos. Em estudos com humanos e animais obesos, o aumento da proteína dietética tem sido eficaz para manter a massa corporal magra (Diez et al., 2002; Vasconcellos et al., 2009).

\section{Metabolismo de carboidratos}

O estudo das respostas glicêmicas e insulínicas dos alimentos se remete, em última instância, ao estudo do metabolismo de carboidratos. Características intrínsecas aos próprios carboidratos, como amidos e fibras, bem como dos alimentos, incluindo composição química e processamento, são importantes. Seu estudo é necessário para cães e gatos, pois parece se relacionar com a manutenção da composição corporal e a incidência de doenças degenerativas (Carciofi, 2007). Alimentos industrializados secos apresentam grande quantidade de carboidratos em sua composição, empregados por questões de processamento de extrusão e custos, havendo importante controvérsia científica sobre suas conseqüências metabólicas para cães (Carciofi et al., 2008) e gatos (De-Oliveira et al., 2008). Este debate foi recentemente posto em voga com a publicação do artigo "The carnivore connection to nutrition in cats” (Zoran, 2002), no entanto parece ter havido certo exagero e ao menos para felinos sadios, os teores de amido normalmente empregados parecem não causar malefício algum.

O controle glicêmico pode estar prejudicado em alguns estágios fisiológicos e condições de saúde, como diabetes melitus, obesidade, gestação, estresse, infecção, câncer e idade avançada (Kahn et al., 2001). Acredita-se que a utilização de dietas que minimizem e estendam a onda 
glicêmica pós-prandial proporciona, para animais nestas condições, o restabelecimento mais rápido e fácil da glicemia (Bouchard \& Sunvold, 1999), aspecto que promete futuros estudos em nutrologia veterinária.

\section{Formulação de macroelementos e urolitíases}

As urolitíases são a causa mais comum de doença obstrutiva do trato urinário inferior em cães e gatos (Wagner et al., 2006). A formação de urólitos no trato urinário é problema clínico significativo para animais de companhia em muitos países (Robertson et al., 2002). Sabe-se que a dieta influencia a composição da urina, de modo que determinados fatores alimentares desempenham papel significativo no aumento do risco ou na prevenção de urolitíases (Stevenson \& Rutgers, 2006).

Atualmente, pesquisadores e empresas buscam a produção de alimentos que atuem na prevenção das urolitíases por estruvita e oxalato de cálcio, os dois tipos mais comuns de urólitos. O desafio na formulação dos alimentos é conciliar as medidas preventivas, já que as mesmas são praticamente opostas em relação a cada um destes urólitos. Em geral, estruvita associa-se a um pH urinário alcalino e oxalato de cálcio a pH urinário ácido, sendo necessário se determinar, por meio da supersaturação urinária, o ponto de equilíbrio entre ambos.

Nos últimos anos foram interessantes os avanços nesta área. Sabe-se hoje que a relação entre os macroelementos que compões a dieta, expressa como o excesso de bases do alimento, apresenta grande correlação com o pH urinário e com equilíbrio ácido-básico de cães e gatos (Zentek \& Schulz, 2004; Jeremias, 2009).

Trabalhos recentes também demonstraram que o $\mathrm{pH}$ urinário, por si só, não permite avaliar o risco de formação de urólitos no trato urinário de cães e gatos. Atualmente, a pesquisa sobre esse tema está focada na determinação da supersaturação relativa, um método que avalia o risco de formação de cristais urinários com base no nível de saturação de sais de fraca solubilidade, tais como o oxalato de cálcio e a estruvita (Allen \& Kruger, 2000). É o método mais utilizado em humanos e foi validado para a urina de cães e de gatos, representando o futuro desta área de investigação (Robertson et al., 2002).

Microbiota do trato gastrintestinal e sua importância na imunidade

O equilíbrio da microbiota intestinal é necessário para as funções digestiva e imunológica dos animais. Saúde comprometida, susceptibilidade às infecções e taxa de crescimento diminuída podem ser sinais de desequilíbrio da microbiota. Estratégias dietéticas para estabelecer e manter o status de eubiose têm incluído o emprego de prebióticos e probióticos (Czarnecki-Maulden, 2008; Carciofi \& Gomes, 2010).

Diferentemente de outros monogástricos e humanos, a pesquisa sobre a microbiota intestinal de cães e gatos é ainda limitada (Hussein \& Sunvold, 2000). No início desta década, ainda havia escassez de informações em animais de companhia sobre o efeito de prebióticos na digestibilidade dos nutrientes, função imunológica intestinal e populações microbianas no intestino grosso (Buddington \& Sunvold, 2000; Swanson et al., 2002).

\section{Considerações Finais}

Nos últimos 10 anos foi importante a modificação ocorrida no direcionamento da pesquisa científica em nutrição de animais de companhia, bem como o avanço do conhecimento nesta área. O modo como cães e gatos estão inseridos na sociedade, a preocupação dos proprietários com alimentação e o desenvolvimento industrial motivado pelo aquecimento econômico do setor, sem dúvida, contribuíram em grande parte para o direcionamento do atual perfil de pesquisas, como foco principal em estudos sobre formulação de alimentos capazes de maximizar a expectativa e a qualidade de vida, pela utilização de ingredientes e nutrientes que desenvolvam a capacidade de resistir à doenças e melhorem a saúde.

Apesar desta evolução, muitos aspectos ainda permanecem desconhecidos na nutrição de cães e gatos, necessitando-se de estudos em caracterização físicoquímica, efeitos de processo, biodisponibilidade e respostas metabólicas dos ingredientes utilizados pela indústria. Paralelo à esta necessidade de informações básicas, a importância e interação da dieta e manejo alimentar com a saúde gastro-intestinal, imunidade e afecções como urolitíases, nefropatias, artropatias, dematopatias, distúrbios cardio-circulatórios, obesidade, diabetes mellitus, dentre outras, também despontam em cenário futuro como horizontes importantes de pesquisa.

\section{Referências}

ALLEN, T.A.; KRUGER, J.M. Enfermedad felina de las vias urinarias. In: HAND, M.S.; THATCHER, C.D.; REMILLARD, R.L. (Eds.) Nutrición clinica en pequeños animales. 4.ed. Bogotá: Panamericana, 2000. p.811-845.

BAUER, J.E. Essential fatty acid metabolism in dogs and cats. Revista Brasileira de Zootecnia, v.37, p.20-27, 2008 (supl. esp.).

BOMTEMPO, V. Nutrition and health of dogs and cats: evolution of petfood. Veterinary Research Communications, v.29, n.2, p.45-50, 2005. 
BOUCHARD, G.F.; SUNVOLD, G.D. Improving canine glycemic response to a meal with dietary starch. In: THE NORTH AMERICAN VETERINARY CONFERENCE, 1999, Orlando. Proceedings... Orlando: The Iams Company Press, 1999. p.16-19.

BOUCHARD, G.F.; SUNVOLD, G.D. Effect of dietary carbohydrate source on postprandial plasma glucose and insulin concentration in cats. In: IAMS NUTRITION SYMPOSIUM, 2., 1998, Wilmington. Proceedings... Wilmington, 2000. p.91-101.

BRUNETTO, M.A.; GOMES, M.O.S.; ANDRE, M.R. et al. Effects of nutritional support on hospital outcome in dogs and cats. Journal of Veterinary Emergency and Critical Care, v.20, p.224-231, 2010.

BRUNETTO, M.A.; PEREIRA NETO, G.B.; TORTOLA, L. et al. Taxas de perda de peso em cães obesos domiciliados e de canil submetidos a um mesmo protocolo de perda de peso. Pesquisa Veterinária Brasileira, v.28, p.26-28, 2008.

BUDSBERG, S.C. Prevention: our best strategy for fighting obesity and its ill effects. Veterinary Medicine, v.104, n.1, p.13, 2010.

CARCIOFI, A.C. Emprego de fibras em alimentos para cães e gatos. In: SIMPÓSIO SOBRE NUTRIÇÃO DE ANIMAIS DE ESTIMAÇÃO, 5., 2005, Campinas. Anais... Campinas: CBNA, 2005, p.95-108.

CARCIOFI, A.C. Métodos para estudo das respostas metabólicas de cães e gatos a diferentes alimentos. Revista Brasileira de Zootecnia, v.36, p.235-249, 2007 (supl. esp.).

CARCIOFI, A.C. Fontes de proteína e carboidratos para cães e gatos. Revista Brasileira de Zootecnia, v.37, p.28-41, 2008 (supl. esp.).

CARCIOFI, A.C.; BAZOLLI, R.S.; BARBUDO, G.R. et al. Efeito de um biscoito extrusado com cobertura de pirofosfato de sódio sobre o cálculo e a placa dentária pré-existentes em cães. ARS Veterinária, v.23, p.47-53, 2007.

CARCIOFI, A.C.; TESHIMA, E.; BAZOLLI, R.S. et al. Qualidade e digestibilidade de alimentos comerciais de diferentes segmentos de mercado para cães adultos. Revista Brasileira de Saúde e Produção Animal, v.10, p.489-500, 2009.

CARCIOFI, A.C.; VASCONCELLOS, R.S.; BORGES, N. et al. Composição nutricional e avaliação de rótulo de rações secas para cães comercializadas em Jaboticabal-SP. Arquivo Brasileiro de Medicina Veterinária e Zootecnia, v.58, p.421-426, 2006

CARCIOFI, A.C.; DE-OLIVEIRA, L.D.; VALÉRIO, A.G. et al. Comparison of micronized whole soybeans to common protein sources in dry dog and cat diets. Animal Feed Science and Technology, v.151, p.251-260, 2009.

CARCIOFI, A.C.; VASCONCELLOS, R.S.; DE-OLIVEIRA, L.D. et al. Chromic oxide as a digestibility marker for dogs-A comparison of methods of analysis. Animal Feed Science and Technology, v.134, p.273-282, 2007.

CARCIOFI, A.C.; GONÇALVES, K.N.V.; VASCONCELLOS, R.S. et al. A weight loss protocol and owners participation in the treatment of canine obesity. Ciência Rural, v.35, p.13311338, 2005

CARCIOFI, A.C.; PONTIERI, R.; FERREIRA, C.F. et al. Avaliação de dietas com diferentes fontes protéicas para cães adultos. Revista Brasileira de Zootecnia, v.35, p.754-760, 2006.

CARCIOFI, A.C.; TAKAKURA, F.S.; DE-OLIVEIRA, L.D. et al. Effects of six carbohydrate sources on dog diet digestibility and post-prandial glucose and insulin response. Journal of Animal Physiology and Animal Nutrition, v.92, p.326-336, 2008.

CARCIOFI, A.C.; GOMES, M.O.S. Dietary effects on gastrointestinal microbiota of aging dogs: potencial tools to health improvement. In: COMPANION ANIMAL NUTRITION SUMMIT - Focus on Gerontology, 2010, Clearwater Beach, Florida. Proceedings... Clearwater Beach: Nestlé Purina, 2010. p.62-69.
CLINE, M.G.; LAUTEN, S.; COX, S. et al. The relationship between obesity and markers of oxidative stress in dogs. Journal of Animal Physiology and Animal Nutrition, v.93, n.2, p.141-142, 2009.

CZARNECKI-MAULDEN, G.L. Effect of dietary modulation of intestinal microbiota on reproduction and early growth. Theriogenology, v.70, p.286-290, 2008.

DE-OLIVEIRA, L.D.; CARCIOFI, A.C.; OLIVEIRA, M.C.C. et al. Effects of six carbohydrate sources on diet digestibility and postprandial glucose and insulin responses in cats. Journal of Animal Science, v.86, p.2237-2246, 2008.

DIEZ, M.; NGUYEN, P.; JEUSETTE, I. et al. Weight loss in obese dogs: evaluation of a high-protein, low-carbohydrate diet. Journal Nutrition, v.132, p.1685S-1687S, 2002.

FAHEY, G.C. Research needs companion animal nutrition. In: KVAMME, J.L.; PHILliPS, T.D. (Eds.) Pet food technology. Mt. Morris: Illinois, 2003. p.135-140.

FORTESA, C.M.L.S.; CARCIOFI, A.C.; SAKOMURA, N.K. et al. Digestibility and metabolizable energy of some carbohydrate sources for dogs. Animal Feed Science and Technology, v.156, p.121-125, 2010.

GERMAN, A.J. The growing problem of obesity in dogs and cats. Journal Nutrition, v.136, p.1940S-1946S, 2006.

HUSSEIN, S.H.; SUNVOLD, G.D. Dietary strategies to decrease dog and cat fecal odor components. In: REINHART, G.A., CAREY, D.P. (Eds.) Recent advances in canine and feline nutrition. Wilmington: Orange Frazer Press, 2000. v.3, p.153168.

JEREMIAS, J.T. Relação entre o excesso de bases do alimento e o pH urinário de gatos. 83f. 2009. Dissertação (Mestrado) - Faculdade de Ciências Agrárias e Veterinárias - Universidade Estadual Paulista, Jaboticabal.

JEREMiAS, J.T.; CARCIOFI, A.C.; BRUnetTO, M.A. et al. Manejo nutricional e digestibilidade no quilotórax canino. Ciência Rural, v.39, p.258-261, 2009

KAHN, S.E.; PRIGEON, R.L.; ROBERT, S.S. et al. Obesity, body fat distribution, insulin sensitivity and islet b-cell function as explanations for metabolic diversity. Journal of Nutrition, v.131, p.354S-360S, 2001

KEALY, R.D.; LAWLER, D.F.; BALLAM, J.M. et al. Effects of diet restriction on life span and age-related changes in dogs. Journal American Veterinary Medical Association, v.220, p.1315-20, 2001.

KRONFELD, D.S. Nature and use of commercial dog foods. Journal American Veterinary Medical Association, v.166, p.487-493, 1975

MICHEL, K.E.; WILLOUGHBY, K.N.; ABOOD, S.K. et al. Attitudes of pet owners toward pet foods and feeding management of cats and dogs. Journal of American Veterinary Medical Association, v.1, n.233, p.1699-703, 2008.

NATIONAL RESEARCH COUNCIL - NRC. Nutrient requirements of dogs. Washington, D.C.: National Academy Science, 1985.

NATIONAL RESEARCH COUNCIL - NRC. Nutrient requirements of cats. Washington: D.C.: National Academy Science, 1986.

NATIONAL RESEARCH COUNCIL - NRC. Nutrient requirements of dogs and cats. Washington, D.C: National Academy Press, 2006.

OLIVEIRA, L.D.; TAKAKURA, F.S.; BAZOLLI, R.S. et al. Dog's and cat's postprandial glycemic and insulinemic responses to different starch souces. In: CONGRESS OF THE EUROPEAN SOCIETY OF VETERINARY AND COMPARATIVE NUtrition, 10., 2006, Nantes. Procedings... Nantes: European Society of Veterinary and Comparative Nutrition, 2006. p.107-107.

ROBERTSON, W.G.; JONES, J.S.; HEATON, M.A. et al. Predicting the crystallization potential of urine from cats and dogs with respect to calcium oxalate and magnesium ammonium 
phosphate (Struvite). Journal of Nutrition, v.132, p.16371641, 2002.

STEIFF, E.L.; BAUER, J.E. Nutritional adequacy of diets formulated for companion animals. Journal of the American Veterinary Medical Association, v.219, n.5, p.601-604, 2001.

STEVENSON, A.; RUTGERS, C. Nutritional management of canine urolithiasis. In: PIBOT, P.; BIOURGE, V.; ELLIOT, D. (Eds). Encyclopedia of canine clinical nutrition. 2006. p.284-315. SWANSON, K.S.; GRIESHOP, C.M.; FLICKINGER, E.A. et al. Supplemental fructooligosaccharides and mannanoligosaccharides influence immune function, ileal and total tract nutrient digestibilities, microbial populations and concentrations of protein catabolites in the large bowel of dogs. Journal of Nutrition, v.132, p.980-989, 2002.

TESHIMA, E.; BRUNETTO, M.A.; VASCONCELLOS, R.S. et al. Nutrient digestibility, but not mineral absorption, is agedependent in cats. Journal of Animal Physiology and Animal Nutrition, DOI: 10.1111/j.1439-0396.2009.00964.x. 2010.

TORTOLA, L.; BRUNETTO, M.A.; ZAINE, L. et al. Uso de psyllium para controle de constipação em cães. Ciência Rural, v.39, p.2638-2641, 2009.

VASCONCELLOS, R.S.; CARCIOFI, A.C.; OLIVEIRA, L.D. et al. Utilização de indicadores para estimar a digestibilidade aparente em gatos. Arquivo Brasileiro de Medicina Veterinária e Zootecnia, v.59, p.466-472, 2007.

VASCONCELLOS, R.S.; CARCIOFI, A.C.; PRADA, F. et al. Avaliação do uso de óxido crômico em cápsulas para estimar a digestibilidade aparente em gatos. ARS Veterinária, v.22, p.4347, 2006.

VASCONCELLOS, R.S.; BORGES, N.C.; GONCALVES, K.N.V. et al. Protein intake during weight loss influences the energy required for weight loss and maintenance in cat. Journal of Nutrition, v.139, p.855-860, 2009.

WAGNER, E.; KEUSCH, C.; IBEN, C. Influence of the feed base excess on urine parameters in cats. Journal of Animal Physiology and Animal Nutrition, v.90, p.19-24, 2006.

YAMKA, R.M.; FRIESEN, K.G.; SCHAKENRAAD, H. The prediction of urine $\mathrm{pH}$ using dietary cations and anions in cats fed dry and wet foods. Intern Journal Appl Research of Veterinary Medicine, v.44, p.58-66, 2006.

ZENTEK, J.; SCHULZ, A. Urinary composition of cats Ia affected by the source of dietary protein. Journal of Nutrition, v.134, p.2162-2165, 2004.

ZORAN, D.L. The carnivore connection to nutrition in cats. Journal American Veterinary Medical Association, v.221, p.1559-67, 2002. 